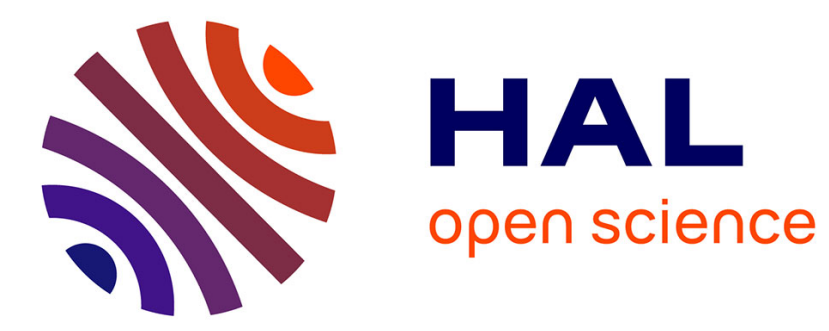

\title{
Initial-state dependence of the route to chaos of an external-cavity laser
}

\author{
A. Locquet, Byungchil Kim, Daeyoung Choi, Nianqiang Li, D S Citrin
}

\section{To cite this version:}

A. Locquet, Byungchil Kim, Daeyoung Choi, Nianqiang Li, D S Citrin. Initial-state dependence of the route to chaos of an external-cavity laser. Physical Review A, 2017, 95 (2), 10.1103/physreva.95.023801. hal-02993514

\section{HAL Id: hal-02993514 https://hal.science/hal-02993514}

Submitted on 6 Nov 2020

HAL is a multi-disciplinary open access archive for the deposit and dissemination of scientific research documents, whether they are published or not. The documents may come from teaching and research institutions in France or abroad, or from public or private research centers.
L'archive ouverte pluridisciplinaire HAL, est destinée au dépôt et à la diffusion de documents scientifiques de niveau recherche, publiés ou non, émanant des établissements d'enseignement et de recherche français ou étrangers, des laboratoires publics ou privés. 


\title{
Initial-state dependence of the route to chaos of an external-cavity laser
}

\author{
A. Locquet, ${ }^{1,2}$ Byungchil Kim, ${ }^{1,2}$ Daeyoung Choi, ${ }^{1,2}$ Nianqiang Li, ${ }^{3}$ and D. S. Citrin ${ }^{1,2}$ \\ ${ }^{1}$ UMI 2958 Georgia Tech-CNRS, Georgia Tech Lorraine, 2 Rue Marconi, F-57070 Metz, France \\ ${ }^{2}$ School of Electrical and Computer Engineering, Georgia Institute of Technology, Atlanta, Georgia 30332-0250, USA \\ ${ }^{3}$ School of Computer Science and Electronic Engineering, University of Essex, Wivenhoe Park, Colchester CO4 3SQ, United Kingdom
}

(Received 28 July 2016; published 1 February 2017)

\begin{abstract}
External-cavity semiconductor lasers (ECLs), consisting of a laser diode in front of a mirror to reflect light back into the laser diode, are among the most important dynamical systems because of their ultrafast dynamics, their tunability, and the numerous existing applications. The dynamics of an ECL is greatly influenced by the existence and stability of numerous modes of the external cavity. In such high-dimensional nonlinear systems, numerous attractors, located around various modes of the external cavity, can coexist in phase space for a given set of parameters, a phenomenon called generalized multistability. In this work, we propose a procedure that allows one to select experimentally different modes of the external cavity as different initial states. We use this procedure to reveal experimentally generalized multistability in an ECL through the demonstration that different routes to chaos exist in an ECL, depending on the initial state selected. In particular, we show that the famous quasiperiodic route to chaos is only observed for specific choices of initial conditions.
\end{abstract}

DOI: 10.1103/PhysRevA.95.023801

\section{INTRODUCTION}

It is well known that dynamical systems, and especially high-dimensional ones, can exhibit generalized multistability [1-3] where several attractors, each with a specific basin of attraction, coexist for given parameters. Indirect evidence of generalized multistability was obtained experimentally in $\mathrm{CO}_{2}$ [4] and semiconductor lasers with filtered [5] or unfiltered [6] optical feedback, by observing hysteresis where different dynamical states are reached when ramping up or down a system parameter, while direct observation of different states was obtained in Ref. [7] in the case of a laser diode subjected to optoelectronic feedback.

External-cavity lasers (ECLs) are a test bench for many conjectures in nonlinear dynamics. They use an external cavity to provide time-delayed optical feedback into the laser diode (LD) and lead to a variety of dynamical behaviors depending on the operating and device parameters. Due to the peculiarities of the delayed feedback, an infinite-dimensional dynamical system results in which hundreds of attractors or attractor ruins can coexist in the same region of phase space, a phenomenon known as generalized multistability and leading to a wealth of dynamical regimes of varying complexity [8-11]. ECLs typically serve as a prototype for high-speed (subnanosecond) chaos in high-dimensional time-delayed dynamical systems [12-15]. Their highly complex dynamics further makes them attractive for applications such as secure communications [16], high-speed random-number generation [17], neuromorphic computing [18], and compressive sensing [19].

Despite interest in ECLs, experiment has suffered, as discussed in Ref. [20], from a lack of detailed and systematic experimental knowledge of the dynamical regimes that can be accessed by a continuous tuning of the operating parameters. Previous experimental results have reported mainly quasiperiodic [13] or period-doubling [21] routes to chaos depending on whether the frequency of the relaxation oscillations $f_{\mathrm{RO}}$ and the free spectral range of the external cavity $f_{\tau}=1 / \tau$ are rationally or irrationally related. These results were based on the observation of a succession of dynamical regimes for a few discrete parameter values.
On the contrary, our work [20] at relatively high current $I$ of $(2-3) I_{\mathrm{th}}$, with $I_{\mathrm{th}}$ the LD threshold current, has resolved an experimental bifurcation of an ECL obtained through the continuous tuning of the feedback level $\eta$. We observed a quasiperiodic route to chaos, involving a primary and secondary Hopf bifurcation, as well as windows of periodicity, before attractor merging and the regime of so-called fully developed coherence collapse (CC) occurs.

In this paper, we report direct evidence of generalized multistability as, for a given set of internal and operating parameters, and in particular for a given ratio between $f_{\mathrm{RO}}$ and $f_{\tau}$, we observe different routes to fully developed chaos, depending on the initial state selected, and characterize their different bifurcation diagrams (BDs).

While coexisting attractors and generalized multistability are expected theoretically, in an experiment it could be assumed that the initial condition (IC) would have little effect on the $\mathrm{BD}$, since one might suppose that noise would cause the trajectory to converge rapidly to the same attractor regardless of ICs. In the context of ECLs, spontaneous-emission noise can make it impractical to select ICs. We have found that selection of different ICs is possible at high $I$ where the output is dominated by stimulated emission, whereas spontaneous emission, the presumed source of noise in careful experiments, plays a smaller role. Specifically, we report a dependence of the route to chaos in a distributed-feedback (DFB) ECL biased at $I \sim(2-3) I_{\text {th }}$ in the long-cavity $L$ case, i.e., in which $L$ is chosen such that the relaxation-oscillation frequency $f_{\mathrm{RO}}>f_{\tau}=\tau^{-1}$. To do so, we select the initial external-cavity mode (ECM) on which the ECL operates at low $\eta$ using a procedure described hereafter.

\section{EXPERIMENTAL RESULTS}

The setup is represented in Fig. 1(a) [22]. The laser used is a single-mode InGaAsP multi-quantum-well DFB laser diode (Mitsubishi ML925B11F) emitting around $1550 \mathrm{~nm}$. The DFB nature of the laser diode ensures single-longitudinal-mode operation, even in the presence of external feedback. An 

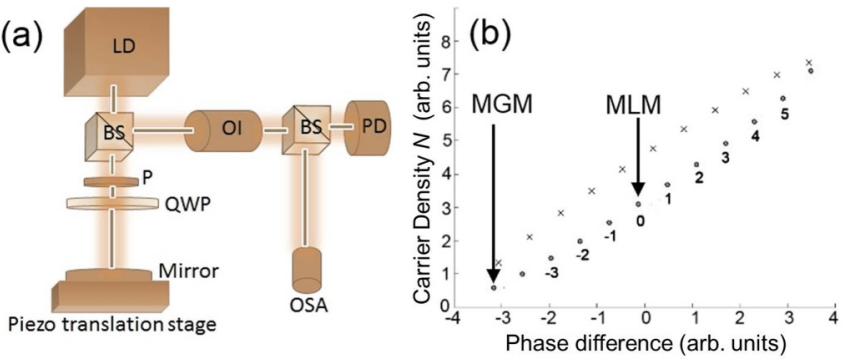

FIG. 1. (a) Diagram of experiment: LD, laser diode; BS, beam splitter; PD, photodetector; $\mathrm{P}$, polarizer; QWP, quarter-wave plate; OI, optical isolator; OSA, optical spectrum analyzer. (b) Ellipse structure of fixed points in the plane of phase difference vs $N$. Circles represent ECMs; crosses represent unstable antimodes. The labels indicate the mode number. Positively numbered modes have a positive shift in frequency with respect to that of the solitary laser; negatively numbered modes have a negative shift in frequency with respect to that of the solitary laser.

oscilloscope captures the optical intensity $\mathcal{I}(t)$. We measure the rf spectrum of $\mathcal{I}(t)$ with a spectrum analyzer and the optical spectrum with a high-resolution optical spectrum analyzer (OSA). We choose $L=30 \mathrm{~cm}$ ( $\tau=2 \mathrm{~ns}$ ) satisfying the long- $L$ criterion; $\eta$, controlled by the reflection in the external cavity (EC) that propagates back into the $\mathrm{LD}$, is varied via the angle of the quarter-wave plate (QWP). The angle is modified thanks to a motorized rotation stage (Thorlabs PRM1Z8) with a trapezoidal velocity profile. To ensure the repeatability of the results, a small velocity of $0.004 \mathrm{rad} / \mathrm{s}$ is used when ramping the angle up or down. Maximum feedback $(\eta=1)$ is reached when the QWP angle is such that the electric field is not subjected to any rotation. In that case, $\sim 20 \%$ of the optical power is fed back on the collimating lens. The optical intensity $\mathcal{I}(t)$, the rf spectrum, the optical spectrum, and the time-averaged voltage $V_{\mathrm{LD}}$ across the injection terminals are used to analyze the dynamical regimes.

Delayed optical feedback is known to have a large impact on ECL dynamics and to lead to two types of steady-state modes: ECMs, separated in frequency by approximately $f_{\tau}$, and unstable antimodes [12]. The Lang-Kobayashi (LK) model [23] gives that the ECMs and antimodes lie on an ellipse in the carrier-density $N$ versus phase-difference $\Delta \phi(t)=$ $\phi(t)-\phi(t-\tau)$ plane, as represented in Fig. 1(b) [24]. The maximum gain mode (MGM) has the the lowest frequency and is usually stable [12,25]. The minimum linewidth mode (MLM) is the closest ECM in frequency to the solitary laser diode mode. The evolution of the system can be visualized in terms of the trajectory moving among the ECMs and antimodes lying on this ellipse. As $\eta$ is increased, some of the ECMs typically undergo bifurcations, turning them into periodic, quasiperiodic, or chaotic attractors localized near the ECM from which they originate. Attractor merging crises can cause a system trajectory to wander among several chaotic limit sets originating from different ECMs, a phenomenon called chaotic itinerancy [9].

In previous work [20], we showed that the forward BD, obtained by ramping up $\eta$, exhibits a complex route to fully developed CC interrupted by windows of periodic and quasiperiodic behaviors, as well as period doubling and

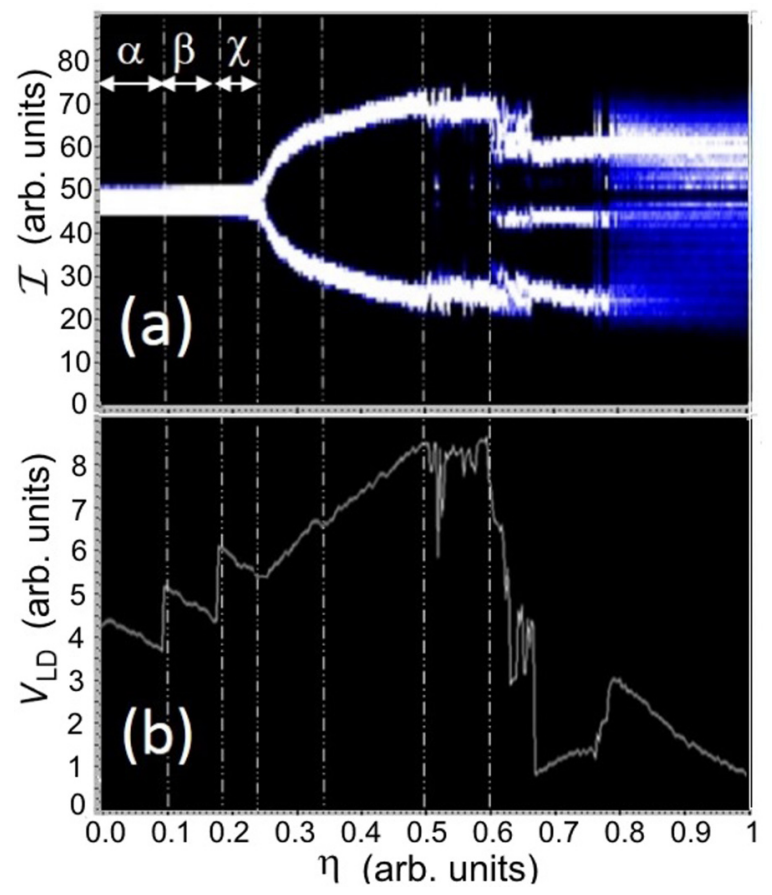

FIG. 2. (a) Reverse $\mathrm{BD}$ and (b) corresponding $V_{\mathrm{LD}} . \quad I=$ $22.59 \mathrm{~mA}$ and $L=30 \mathrm{~cm}$. $I_{\mathrm{th}}=9.27 \mathrm{~mA}$.

intermittency. At low $\eta$ and high $I$, the participating ECMs appear with a positive frequency shift with respect to the solitary laser diode, while at high $\eta$, near $\mathrm{CC}$, the optical spectrum shows negative-frequency-shifted ECMs.

One can freely choose ICs in simulations; in experiment, IC selection is challenging. We introduce a controlled way to select the IC by exploiting dynamical hysteresis. In a forward BD starting at $\eta=0$, the ECL initially lases on the MLM (ECM 0) and then undergoes a sequence of bifurcations leading to a given $\mathrm{BD}$, described later. Once this forward $\mathrm{BD}$ is obtained, we map out the reverse BD starting from $\eta=1$ and ramping it down. The resulting reverse $\mathrm{BD}$, which displays significant differences with the forward BD, is shown in Fig. 2(a) and the corresponding $V_{\mathrm{LD}}$ is shown in Fig. 2(b). It has been shown $[11,26,27]$ that $V_{\mathrm{LD}}$ tracks the changes in the time-averaged $N$. One observes two discontinuities in $V_{\mathrm{LD}}$ at $\eta=0.1$ and 0.18 [Fig. 2(b)] in the horizontal band toward the left of the BD, corresponding to $\mathrm{cw}$ output by the LD. These discontinuities reveal that different ECMs are participating in the ECL dynamics in regions $\alpha, \beta$, and $\chi$ of Fig. 2(a). Specifically, the analysis of the optical spectrum reveals that, as $\eta$ decreases from 0.24 to 0 , the first discontinuity in $V_{\mathrm{LD}}$ corresponds to a shift from ECM 2 to ECM 1, while the second discontinuity corresponds to a shift from ECM 1 to ECM 0: Fig. 4(a) corresponds to Fig. 2, $\alpha$; Fig. 5(a) to Fig. 2, $\beta$; and Fig. 6(a) to Fig. 2, $\chi$.

Stopping the reverse BD at a feedback level that lies in region $\beta$ or $\chi$ of Fig. 2(a) thus allows one to access two additional ECMs, ECM 1 and 2, respectively. Increasing the feedback level from the value reached in regions $\alpha, \beta$, and $\chi$ then leads to three different forward BDs starting from the three different ECMs (0, 1, and 2), represented in Figs. 3(a), 3(b), and $3(\mathrm{c})$, respectively. It must be noted that, even though 


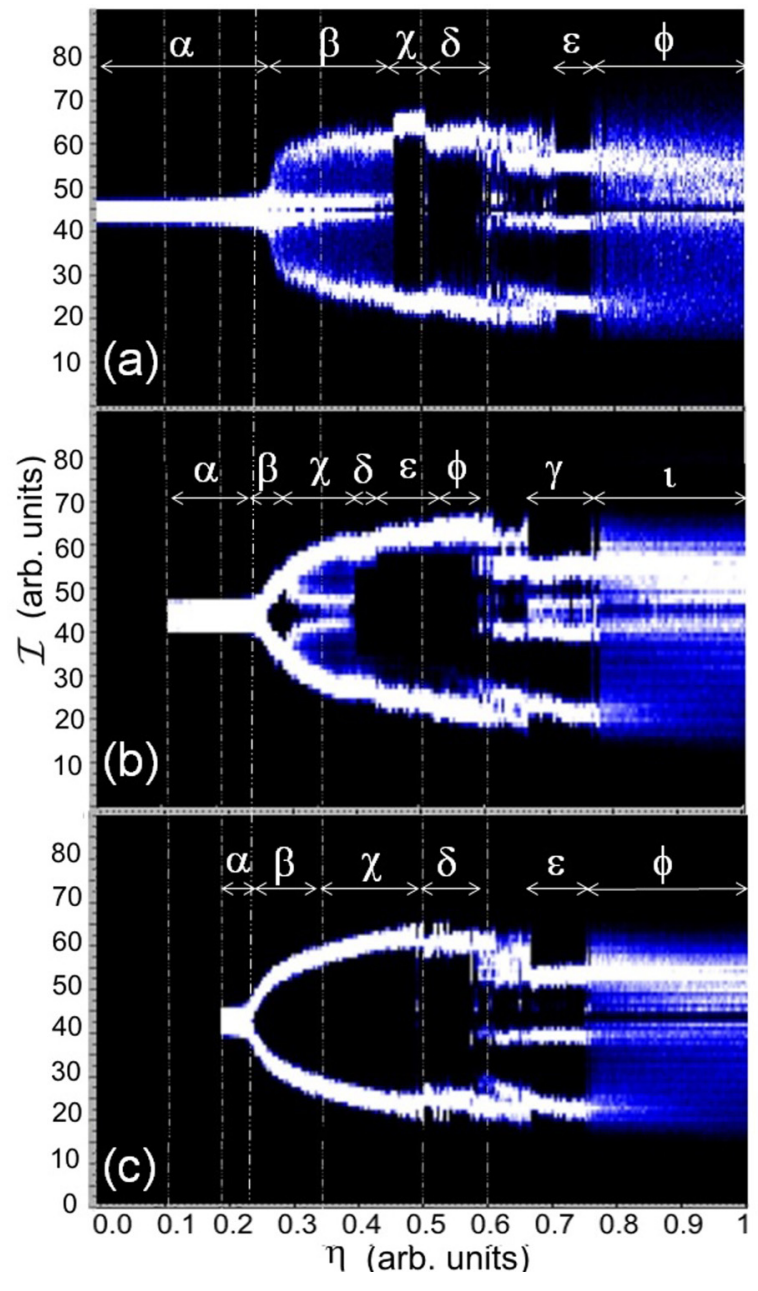

FIG. 3. Forward BDs with $I=22.59 \mathrm{~mA}$ and $L=30 \mathrm{~cm}$. Initial mode (a) ECM 0, (b) ECM 1, and (c) ECM 2.

the lowest $\eta$ reached in the three BDs is different, the three BDs overlap for $\eta$ in the interval $[0.18,1]$. As a result, in the interval $[0.18,1]$, all system parameters are identical except for the initial state of the ECL at $\eta=0.18$. The three BDs thus represent three different routes to fully developed $\mathrm{CC}$ of the same ECL starting from three different initial states. The difference among the three BDs starting from different ECMs, shown in Fig. 3, is shown below to be significant in the sense that the route to chaos indeed depends on the ICs. Moreover, this difference confirms the coexistence of several attractors sharing different basins.

Figure 3(a) shows the forward BD starting from ECM 0, and Fig. 4 shows the corresponding optical spectra. It must be noted that ECM 0 is the mode on which the laser lies naturally when a small amount of feedback is added to a LD. As a consequence, the BD of Fig. 3(a) is also the typical BD that one obtains by applying increasing levels of feedback $\eta$ to an initially quiescent LD. Moreover, we observe clearly from the optical spectrum that in the entire region $\alpha$ of Fig. 3(a), i.e., over an interval $\eta<0.24$, only one ECM (0) of the solitary laser (MLM) participates in the cw dynamics. This is strikingly different from the observed dynamics in the reverse BD (Fig. 2), where three different ECMs participate in the
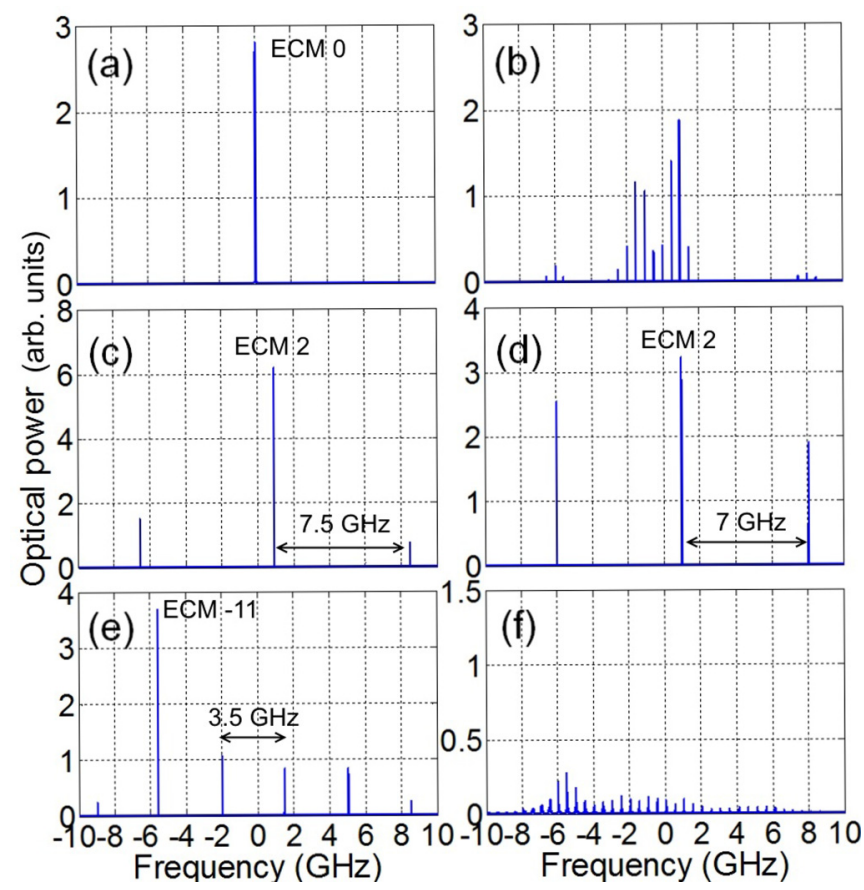

FIG. 4. Optical spectrum with the IC ECM 0: (a) $\eta=0$, (b) $\eta=$ 0.35 , (c) $\eta=0.49$, (d) $\eta=0.55$, (e) $\eta=0.75$, and (f) $\eta=0.9$.

dynamics, in the same range of $\eta$. This observation highlights the role of the reverse BD that constrains the system trajectory to end up in the basin of the stable equilibria corresponding to ECMs 1 and 2.

When $\eta>0.24$, the optical spectrum [Fig. 4(b)] shows that several ECMs ( -3 to 3 ) participate in the dynamics. An analysis of $\mathcal{I}(t)$ (not shown here) shows the corresponding temporal dynamics is quasiperiodic.

Further increasing $\eta(\sim 0.47)$, a discontinuity appears in the BD $[\chi$ of Fig. 3(a)] corresponding to an abrupt change of the dynamics to a limit cycle. The optical spectrum [Fig. 4(c)] reveals the oscillation now occurs around ECM 2, and that its frequency, 7.5 GHz, is close to $f_{\mathrm{RO}}$.

Further increasing $\eta$, at $\sim 0.5$, another limit cycle at $f_{\mathrm{RO}} \sim$ $7 \mathrm{GHz}$ is seen [ $\delta$ of Fig. 3(a)], still around ECM 2, as seen in Fig. 4(d). Previous works $[13,28,29]$ show that our observation of two limit cycles with different frequencies $(7.5$ and $7 \mathrm{GHz}$ ) around the same ECM is compatible with the LK prediction.

Increasing $\eta$ to $\sim 0.6$, intermittency between the dynamics of $\delta$ and $\epsilon$ of Fig. 3(a) is observed. Region $\epsilon$, corresponding to $\eta \gtrsim 0.74$, shows a periodic oscillation with fundamental frequency $\sim 7 \mathrm{GHz}$ and a subharmonic at $\sim 3.5 \mathrm{GHz}$, around $\mathrm{ECM}-11$ [Fig. 4(e)]. The harmonic indicates that the $3.5-\mathrm{GHz}$ limit cycle likely originates from a period-doubling bifurcation of a $7-\mathrm{GHz}$ cycle that must have existed, at lower $\eta$, near $\mathrm{ECM}-11$. If $\eta$ is further increased, the limit cycle disappears, leading to $\mathrm{CC}(\phi)$ and involving multiple ECMs negatively shifted in frequency [Fig. 4(f)] with respect to the MLM.

The forward BD starting from ECM 1 is shown in Fig. 3(b), and Fig. 5 shows the corresponding optical spectrum. The ECL initially displays cw behavior on ECM 1 [Fig. 5(a); region $\alpha$ ( $\eta \sim 0.22$ ) of Fig. 3(b)]. As $\eta$ exceeds 0.22 , the BD reveals that the ECL undergoes a periodic oscillation $[\beta$ of Fig. 3(b)]. This 

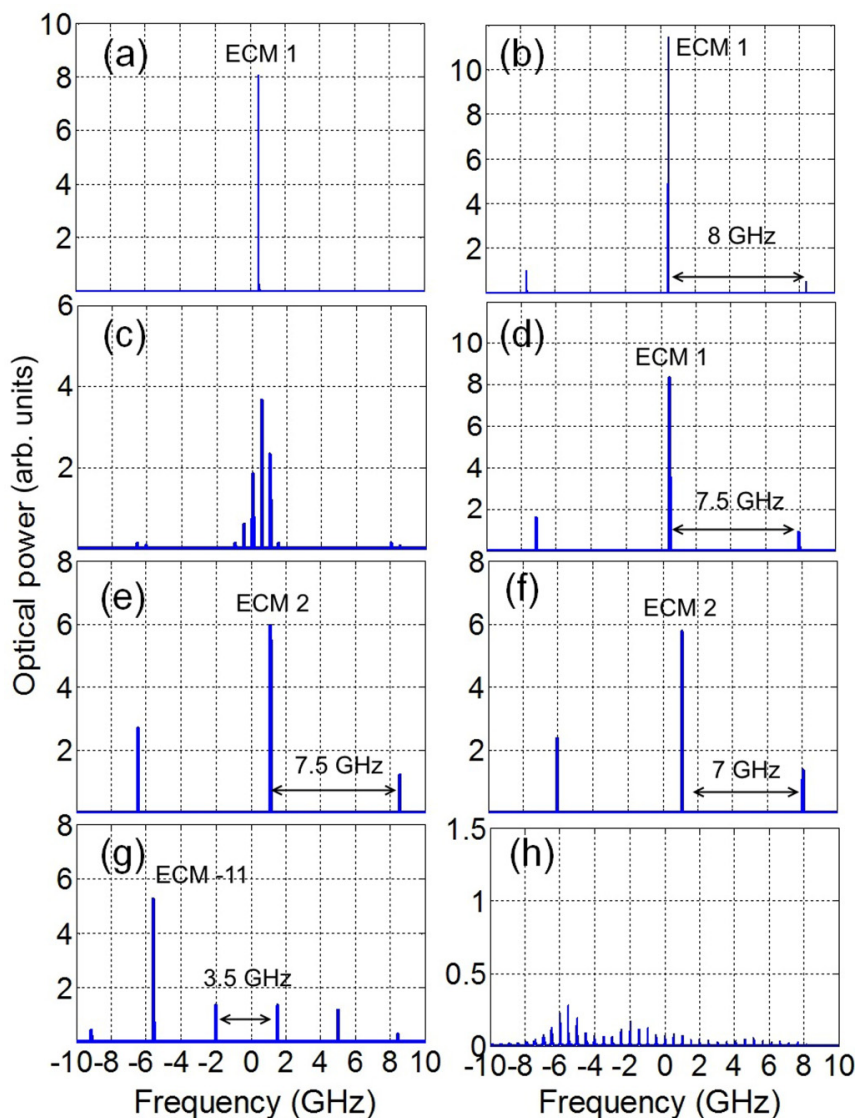

FIG. 5. Optical spectrum with the IC ECM 1: (a) $\eta=0.15$, (b) $\eta=0.24$, (c) $\eta=0.35$, (d) $\eta=0.42$, (e) $\eta=0.47$, (f) $\eta=0.55$, (g) $\eta=0.75$, and (h) $\eta=0.9$.

oscillation is manifested as sidebands $\pm 8 \mathrm{GHz}$ from ECM 1 [Fig. 5(b)]. This transition in the dynamics corresponds to a Hopf bifurcation leading to a limit cycle with frequency close to $f_{\mathrm{RO}}$, consistently with the predictions of the LK model $[13,30]$. With increasing $\eta$, additional sidebands near $\pm f_{\tau}$ appear in the optical spectrum [Fig. 5(c)], showing a second frequency in the dynamics [ $\chi$ of Fig. 3(b)]. The undamping of a second frequency near $f_{\tau}$ corresponds to a second Hopf bifurcation and signals the development, in phase space, of a torus attractor. Note that both the limit cycle and the torus result from the destabilization of ECM 1 and are located around it in phase space [Figs. 5(b) and 5(c)].

Further raising $\eta$, the dynamics becomes periodic again [ $\delta$ of Fig. 3(b)]. The optical spectrum shows [Fig. 5(d)] this cycle is still centered on ECM 1 with a frequency of $\sim 7.5 \mathrm{GHz}$. Since the dominant ECM 1 does not change between Figs. 5(b) and 5(d), we have here evidence of the existence, at different $\eta$, but around the same ECM (1), of two periodic solutions with different frequencies, 8 and $7.5 \mathrm{GHz}$, with separation $\sim f_{\tau}$.

As $\eta$ is increased, and region $\epsilon$ of Fig. 3(b) is reached, a small discontinuity is seen in the BD. The optical spectrum [Fig. 5(e)] reveals that this discontinuity corresponds to a shift from ECM 1 to ECM 2. The ECL still oscillates periodically, and the optical spectrum confirms that the frequency is still 7.5 GHz. We have thus observed a transition between two

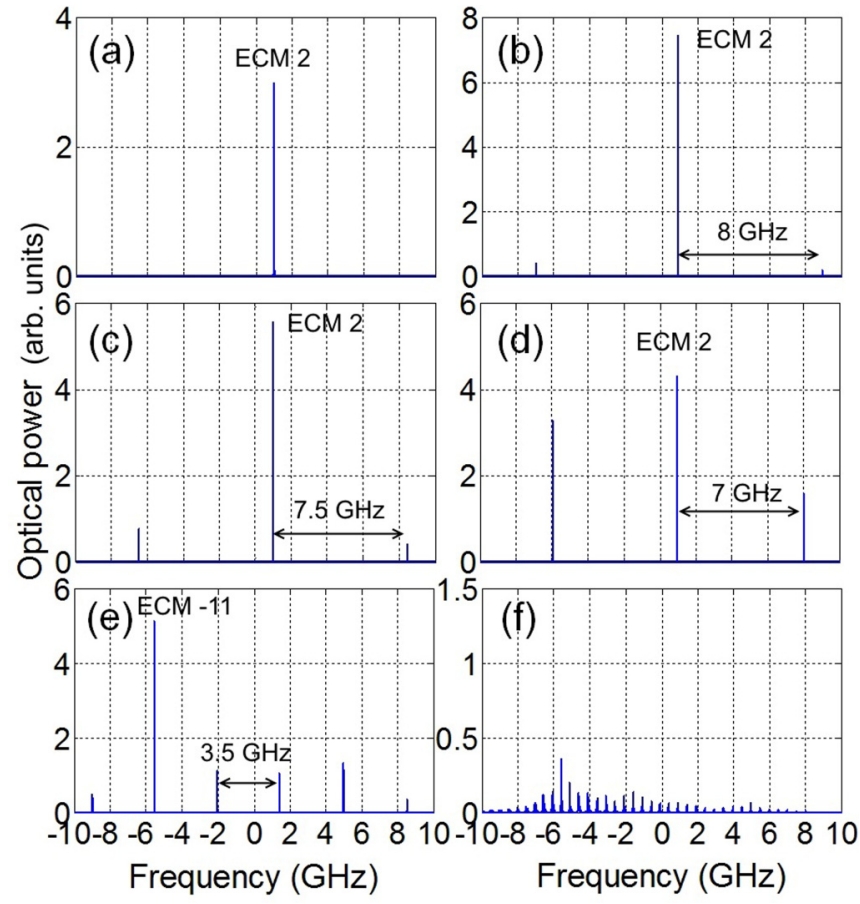

FIG. 6. Optical spectrum with the IC ECM 2: (a) $\eta=0.2$, (b) $\eta=0.3$, (c) $\eta=0.39$, (d) $\eta=0.55$, (e) $\eta=0.7$, and (f) $\eta=0.9$.

limit cycles of the same period, but they developed around two different, neighboring, modes of the ECL. Increasing $\eta$ further, the scenario is similar to that in Fig. 3(a): the trajectory moves to a limit cycle of frequency $\sim 7 \mathrm{GHz}$ around ECM $2[\phi$ of Fig. 3(b) and Fig. 5(f)]. Then the trajectory moves toward negatively shifted ECMs: first a period-doubled limit cycle around ECM - 11 is seen $(\gamma)$, then a regime of fully developed CC $(\iota)$.

Figure 3(c) shows the forward BD starting from ECM 2 and Fig. 6, the optical spectrum. In the initial region $\alpha(\eta \gtrsim 0.22)$ of Fig. 3(c), the ECL displays cw behavior located on ECM 2 [Fig. 6(a)]. As $\eta$ is raised above 0.22 and region $\beta$ of Fig. 3(c) is reached, the ECL undergoes a periodic oscillation [Fig. 6(b)], seen as sidebands $\pm 8 \mathrm{GHz}$ away from ECM 2. Increasing $\eta$ further, the analysis of the optical spectrum reveals the existence of two additional distinct regions $\chi$ and $\delta$ in Fig. 3(c), corresponding to the appearance of two other limit cycles. Both limit cycles are centered on ECM 2; the one in region $\chi$ has a frequency of $\sim 7.5 \mathrm{GHz}$ [Fig. 6(c)] while the one in region $\delta$ of $\sim 7 \mathrm{GHz}$ [Fig. 6(d)]. It is remarkable that the active ECM (2) does not change between Figs. 6(b), 6(c), and 6(d), therefore revealing experimentally the existence, at different $\eta$, of three periodic solutions with frequencies $8,7.5$, and $7 \mathrm{GHz}$, and thus with separation $\sim f_{\tau}$, around the same ECM. Finally, for larger $\eta$, the BD is similar to that of the two previous cases.

\section{CONCLUSION}

Our results constitute direct evidence of generalized multistability in an ECL. In particular, we observe the existence, for a given set of internal and operating parameters, of different routes to $\mathrm{CC}$, depending on the initial state selected. Specifically, it is remarkable that, despite the fact that in our experiment $f_{\mathrm{RO}}$ and $f_{\tau}$ are incommensurate, a true 
quasiperiodic route to chaos, involving the development of a limit cycle and then a torus attractor around a given ECM, as expected from the literature [31], is not systematically observed (e.g., when the initial state is set to be around ECM 2). The BDs reveal that the choice of the initial state is especially important for small levels of feedback at which several stable attractors, with separate basins, and with a small positive shift in frequency with respect to the solitary laser, are observed, confirming earlier predictions [12,31]. As the feedback level is increased, the observed dynamics tends to move toward ECMs with greater positive frequency shift, owing probably to their larger basins [12]. Several limit cycles, with frequencies close to $f_{\mathrm{RO}}$, but differing by about $f_{\tau}$, are typically involved in this transition. The different BDs converge though on a limit cycle oscillating at $\sim f_{\text {RO }}$, after which a further increase of the feedback level
( $\eta \gtrsim 0.5$ ) leads to the same dynamical regimes, involving periodicity, intermittency, period doubling, and finally chaotic itinerancy among numerous ECMs that are negatively shifted in frequency with respect to the solitary laser, as is typically reported in the literature [31]. In conclusion, our results provide a selection method for ECMs and make use of it to reveal generalized multistability in an ECL through the demonstration of different routes to chaos that depend on the initial state selected.

\section{ACKNOWLEDGMENTS}

The authors acknowledge the support of the Conseil Régional de Lorraine, of the Centre National de la Recherche Scientifique, and of the School of Electrical and Computer Engineering of the Georgia Institute of Technology.
[1] F. T. Arecchi, R. Meucci, G. Puccioni, and J. Tredicce, Phys. Rev. Lett. 49, 1217 (1982).

[2] E. Eschenazi, H. G. Solari, and R. Gilmore, Phys. Rev. A 39, 2609 (1989).

[3] J. M. Saucedo-Solorio, A. N. Pisarchik, A. V. Kir'yanov, and V. Aboites, J. Opt. Soc. Am. B 20, 490 (2003).

[4] F. T. Arecchi, G. Giacomelli, A. Lapucci, and R. Meucci, Phys. Rev. A 43, 4997 (1991).

[5] A. Fischer, O. Andersen, M. Yousefi, S. Stolte, and D. Lenstra, IEEE J. Quantum Electron. 36, 375 (2000).

[6] B. Kim, A. Locquet, N. Li, D. Choi, and D. Citrin, IEEE J. Quantum Electron. 50, 965 (2014).

[7] G.-Q. Xia, S.-C. Chan, and J.-M. Liu, Opt. Express 15, 572 (2007).

[8] M. C. Soriano, J. J. Garca-Ojalvo, C. R. Mirasso, and I. Fischer, Rev. Mod. Phys. 85, 421 (2013).

[9] J. Ohtsubo, Semiconductor Lasers: Stability, Instability and Chaos (Springer, New York, 2006).

[10] A. Prasad, Y.-C. Lai, A. Gavrielides, and V. Kovanis, Phys. Lett. A 314, 44 (2003).

[11] D. Brunner, M. C. Soriano, X. Porte, and I. Fischer, Phys. Rev. Lett. 115, 053901 (2015).

[12] C. Masoller and N. B. Abraham, Phys. Rev. A 57, 1313 (1998).

[13] J. Mork, J. Mark, and B. Tromborg, Phys. Rev. Lett. 65, 1999 (1990).

[14] G. H. M. van Tartwijk and G. P. Agrawal, Prog. Quantum Electron. 22, 43 (1998).

[15] D. Brunner, X. Porte, M. C. Soriano, and I. Fischer, Sci. Rep. 2, 732 (2012).
[16] A. Argyris, D. Syvridis, L. Larger, V. Annovazzi-Lodi, P. Colet, I. Fischer, J. García-Ojalvo, C. R. Mirasso, L. Pesquera, and K. A. Shore, Nature (London) 438, 343 (2005).

[17] A. Uchida, K. Amano, M. Inoue, K. Hirano, S. Naito, H. Someya, I. Oowada, T. Kurashige, M. Shiki, S. Yoshimori, K. Yoshimura, and P. Davis, Nat. Photon. 2, 728 (2008).

[18] D. Brunner et al., Nat. Commun. 4, 1364 (2013).

[19] D. Rontani, D. Choi, C. Y. Chang, A. Locquet, and D. S. Citrin, Sci. Rep. 6, 35206 (2016).

[20] B. Kim, A. Locquet, D. Choi, and D. S. Citrin, Phys. Rev. A 91, 061802 (2015).

[21] H. Li et al., IEEE J. Quantum Electron. 29, 2421 (1993).

[22] B. Kim, N. Li, A. Locquet, and D. S. Citrin, Opt. Express 22, 2348 (2014).

[23] R. Lang and K. Kobayashi, IEEE J. Quantum Electron. 16, 347 (1980)

[24] C. H. Henry and R. F. Kazarinov, IEEE J. Quantum Electron. 22, 294 (1986).

[25] T. Heil, I. Fischer, and W. Elsäßer, Phys. Rev. A 58, R2672(R) (1998).

[26] W. Ray, W.-S. Lam, P. N. Guzdar, and R. Roy, Phys. Rev. E 73, 026219 (2006).

[27] A. A. Sahai, B. Kim, D. Choi, A. Locquet, and D. S. Citrin, Opt. Lett. 39, 5630 (2014).

[28] A. Ritter and H. Haug, IEEE J. Quantum Electron. 29, 1064 (1993).

[29] T. Erneux, Proc. SPIE 3944, 588 (2000).

[30] J. Ohtsubo, Opt. Rev. 6, 1 (1999).

[31] J. Mork, B. Tromborg, and J. Mark, IEEE J. Quantum Electron. 28, 93 (1992). 